\title{
Violating the second law of thermodynamics in a dynamical system through equivalence closure via mutual information carriers of a 5-tuple measure space
}

This paper was downloaded from TechRxiv (https://www.techrxiv.org).

\section{LICENSE}

CC BY 4.0

SUBMISSION DATE / POSTED DATE

22-10-2021 / 26-10-2021

\section{CITATION}

Bhattacharjee, Deep (2021): Violating the second law of thermodynamics in a dynamical system through equivalence closure via mutual information carriers of a 5-tuple measure space. TechRxiv. Preprint. https://doi.org/10.36227/techrxiv.16854772.v1

$\mathrm{DOI}$ 


\title{
Violating the second law of thermodynamics in a dynamical system through equivalence closure via mutual information carriers of a 5-tuple measure space
}

\author{
Deep Bhattacharjee ${ }^{1,2}$ \\ ${ }^{I}$ Project Director of AATWRI - R\&D Directorate of Electro-gravitational Simulation \& Propulsion \\ Laboratory, Bhubeneshwar, Odissa, India \\ ${ }^{2}$ Database Administrator at PhyxBuzz, Kolkata, West Bengal, India \\ Email: itsdeep@live.com, hi@phyxbuzz.com
}

\begin{abstract}
Time and space average of an ergodic systems following the 5-tuple relations $(\mathcal{A}, \sim, \mathcal{J}, \Sigma, \mu)$ through the initial increment from $a+b \theta$ to $a+c+b \theta$ indicates the entropy to be reserved in the deterministic yet dynamical and conservative systems to hold for the set $S_{p}=S_{1} \sum_{i=2}^{\infty} S_{i}$ keeping $S$ as the entropy $\exists\left(S_{\infty}=\cdots S_{3}=S_{2}\right)>S_{1}$ obeying the Poincaré recurrence theorem throughout the constant attractor $\mathcal{A}$. This in turn states the facts of the equivalence closure as the property of the induced systems to resemblance an entropy conserving scenario.
\end{abstract}

Keywords - Ergodic theory; Poincaré recurrence theorem; Entropy; Liouville's theorem; Mutual information

Introduction - Periodicity of a system can be defined by the number of periodical iterations, the function is taking place over a period of time. That function in most cases, as has been observed in nature is chaotic making the system totally unpredictable in its future outcomes, but to some extent physicists have been able to determine whether the function with initial bifurcations is making a pattern observable in the final bifurcations. This tendency of a system is natural and the order emerges from the disorder. Thus every chaotic function that initially started making large bifurcations might settle down to a small bifurcations after a repeated interval till again it starts to behave with chaotic bifurcations. The system is then evolving with time and hence dynamical in its own periodicity. It is not necessary that the system is generated and continued with iterations in a confined place, it can randomize to a much more spacious way where some parts may dissipate outwards without returning back. Hence, the property of that function has not been conserved over time. Its time and space measure may give random values at the initial and final state. But there are instances of the non-dissipative functions and that too being a conserved one. Thus the notion of ergodicity been developed 
with a more probably conservative limit that the system generates over a finite period of time. It is nevertheless crucial to determine the nature of such a function where the system is both dynamical and conservative, thus being ergodic. This is the prospects of this paper. Just as, any equation can be plotted as a graph, a special type of equation has been discussed here where there is the capacity of a central attractor around which the points of the equation evolves in accordance with the limits of the variables. This in turn imposes the system to act repeatedly taking the shape over and over without losing its track between the initial and the final states. Thus, the points which are monodromic around the attractor might revolve infinitely taking its initial pattern over and over again. Therefore, one might suggest that, the function itself acts over the space where the entropy is increasing with each iterations, but it has been shown in this paper that, such a case does not exist over the modified Archimedean spiral equations that have been considered in the function space. Therefore, the initial state when jumps to its next periodic state may give rise to many other, rather numerous periodic states without naturally losing any kind of entropy. That being said, the entropy has been conserved throughout the process. Both the case of joint and conditional entropy has been considered while making this paper and it has been deliberately proved that, its only through the equivalence closure, that the function stops its entropy from getting increased over time thus violating the $2^{\text {nd }}$ law of thermodynamics. Saying that, it needs to be considered that, entropy is also the 'quantized' information carriers where the mutual information from one state to another is being conveyed by $\theta$ in the modified equations of $a+c+d+e+\cdots+b \theta$. This mutual information that has been transmitted by the function over the period of time makes the entropy conserved through the repeated iterations. Set theoretic approach of analysis has been provided in this paper to depict the states of the function through a series of five diagrams where the base points $\left(x_{0}, y_{0}\right)$ has been made as the initial pole from where the spiral is generated by a generator that continues up to infinity, provided the values are bounded over a certain interval. It has also been demonstrated that the system loses its track of dynamicity and behaves as a circle that expands and contracts depending on the determinants of the spinner and spreader.

Methodology - To measure the dynamical evolution of a system in a geometric space, think of the system as a particle and space as a state where a typical temporal evolution term $\tau_{p}$ acts on all points $p$ of the space $S_{p}$ 
through both time and space evolution operators that goes on the 5 -tuple relations $(\mathcal{A}, \sim, \mathcal{J}, \Sigma, \mu)$ where each item is defined as $[1]$;

- $\mathcal{A}$ is the attractor of the space of the evolving system where every union takes place surrounding its boundary point $\partial \mathcal{A}_{p}$ defined as $S_{1} \cup S_{2} \cup S_{3} \ldots S_{i}$ in the set $\left\{x \in X \mid x=\underset{\substack{p=0 \\ S_{p} \subset X}}{\infty} X\right\}$ where the initial evolution parameter $S_{1}$ keeps track of all the positions from $S_{2}$ onwards in the space $S_{p}$ thereby conserving entropy over the whole set $X$.

- $\quad \sim$ is the equivalence closure where $\bigsqcup_{i \in I} X_{I} / \sim$ forms the locus of the periodicity that makes the boundary points $\partial \mathcal{A}_{p}$ evolves keeping the entropy conserved with the initial state as the final state.

- $\mathcal{J}$ is the non-empty set where $\mathcal{J} \in \Sigma \exists X \backslash S_{p} \in \Sigma$ that has been closed by countable unions $S_{1} \cup S_{2} \cup$ $S_{3} \ldots S_{i}$ then $S_{p} \in \Sigma$.

- $\quad \Sigma$ is the $\sigma$-algebra on the set $\mathcal{J}$.

- $\mu(\mathcal{J})$ is the Haar measure of the space $(\mathcal{J}, \Sigma)$

A dynamical space that is measure preserving, under a transformation $\tau_{p}: \mathcal{J} \rightarrow \mathcal{J}$ is the probability space such that $[2]$;

$$
\forall \mathcal{J} \in \Sigma, \mu(\mathcal{J})=\mu\left(\tau_{p}(\mathcal{J})\right)
$$

Elaborating this particular transformation $\tau_{p}$; a number of iterations can be taken to develop a total trajectory space as;

$$
\tau_{p}{ }^{-k} \mathcal{J}, \ldots \tau_{p}{ }^{-1} \mathcal{J}, \mathcal{J}, \tau_{p}{ }^{1} \mathcal{J}, \ldots \tau_{p}{ }^{k} \mathcal{J} \in \mathcal{J}
$$

$\tau_{p}$ is then a computable ergodic for the transformation functions in $\mathcal{J}$ with $\Sigma$ as $\tau_{p}{ }^{-1} \mathcal{J}$, either, $\mu(\mathcal{J})=0$ or $\mu(\mathcal{J})=1$. In a ergodic system, the 5 -tuple relations $(\mathcal{A}, \sim, \mathcal{J}, \Sigma, \mu)$ is a measure space where $\xi$ is a $\mu$ integrable function for $\xi \in L^{1}(\mu)$ provided the time average over all iterations $\tau_{p}$ starting from the boundary domain $\partial \mathcal{A}_{p}$ over points from $S_{1}$ stated as [3]; 


$$
\hat{\xi}\left(J_{p}\right)=\lim _{n \rightarrow \infty} \frac{1}{n} \sum_{k=0}^{n-1} \xi\left(\tau_{p}{ }^{k} \mathcal{J}\right)
$$

And if the Haar measure $\mu(\mathcal{J}) \geq 0$ everywhere, the space average can be defined as;

$$
\bar{\xi}=\frac{1}{\mu(\mathcal{J})} \int \xi d \mu, \forall \mu(\mathcal{J})=1
$$

In ergodicity, the time average is equal to space average where for every $\mathcal{J}$ there exists a $\xi$, the function takes place in conjugation with $\hat{\xi} \circ \tau_{p}$ as;

$$
\hat{\xi} \circ \tau_{p}=\hat{\xi} \forall \hat{\xi} \in L^{1}(\mu)
$$

For the normalization to make sense along the trajectory with $\mu(\mathcal{J}) \geq 0$ everywhere, the relation that needs to be satisfied are;

$$
\int \hat{\xi} d \mu=\int \xi d \mu, \forall \bar{\xi}=\hat{\xi}
$$

Thus proving the essence of ergodicity as;

$$
\lim _{n \rightarrow \infty} \frac{1}{n} \sum_{k=0}^{n-1} \xi\left(\tau_{p}{ }^{k} \mathcal{J}\right)=\frac{1}{\mu(\mathcal{J})} \int \xi d \mu
$$

To establish this, we will briefly proceed with a spiral equation starting as $a+b \theta$ with its gradual evolution to $a+c+b \theta$ where the use of the incomplete elliptic integral of the $2^{\text {nd }}$ kind $E(x, k)$ is inevitable through the norm of the space $\left\|S_{p}\right\|$ that is the ensemble of the closed union of the entropy point under the inverse image through $S_{2}$ up to $S_{i}$ where the base points $\left(x_{0}, y_{0}\right) \in S_{1}$ generating the spiral is a quotient space as regards to their smash product provided we take the generators of the space as an ensemble of $i^{\text {th }}$ points of $x$ and $y$ as;

$$
\left(x_{0}, y_{0}\right) \bigotimes\left(x_{i}, y_{i}\right)=\left(x_{0}, y_{0}\right) \times\left(x_{i}, y_{i}\right) \backslash\left(x_{0}, y_{0}\right) \bigvee\left(x_{i}, y_{i}\right)
$$




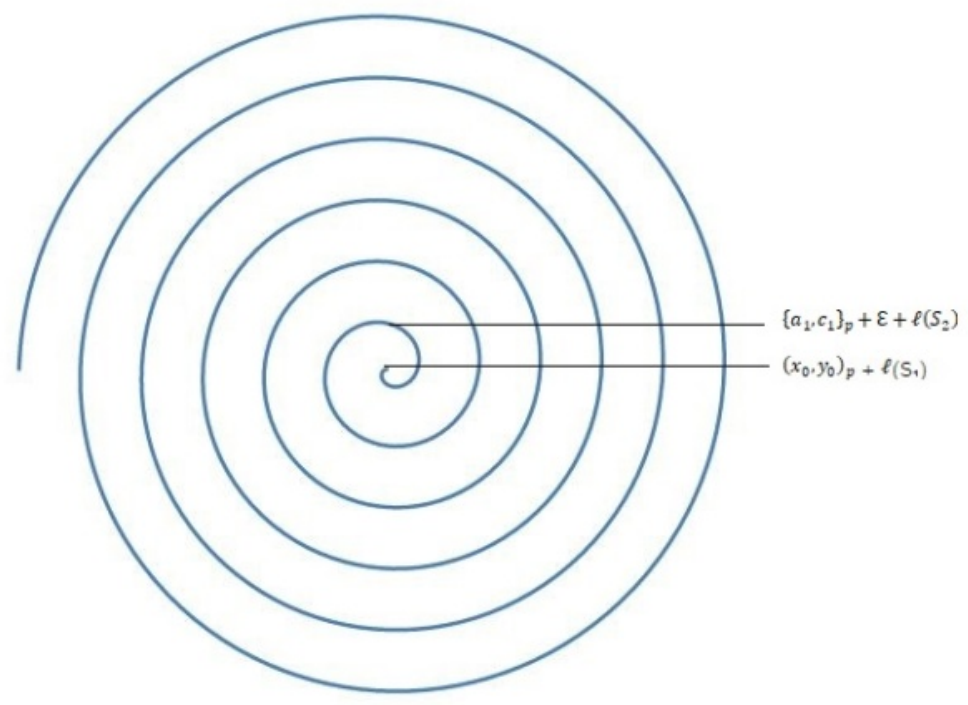

Figure (a)

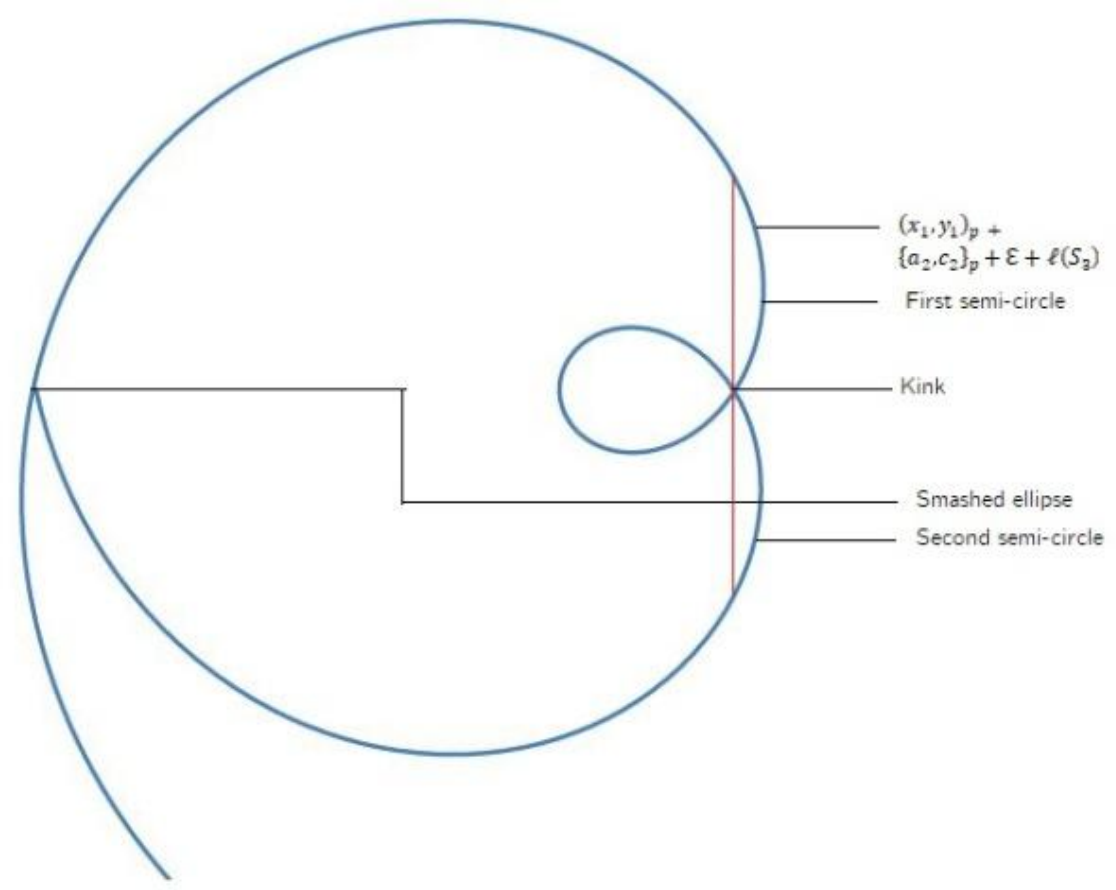

Figure (b) 


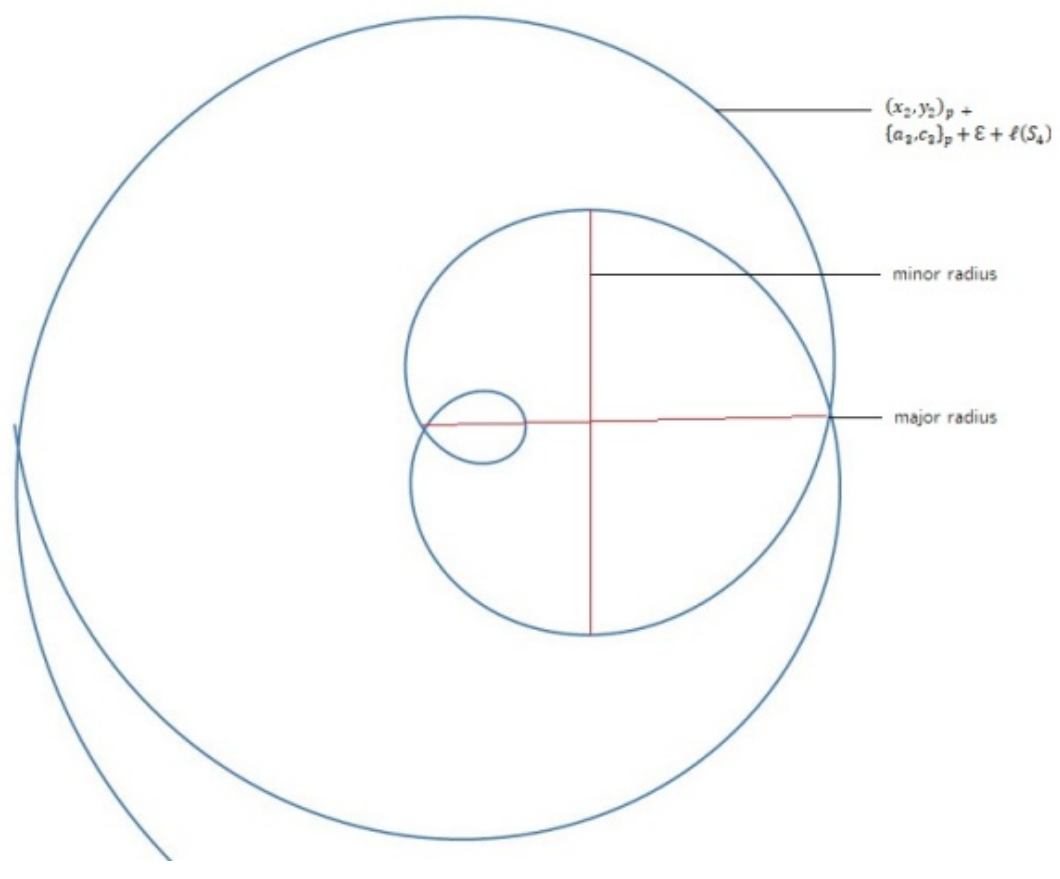

Figure (c)

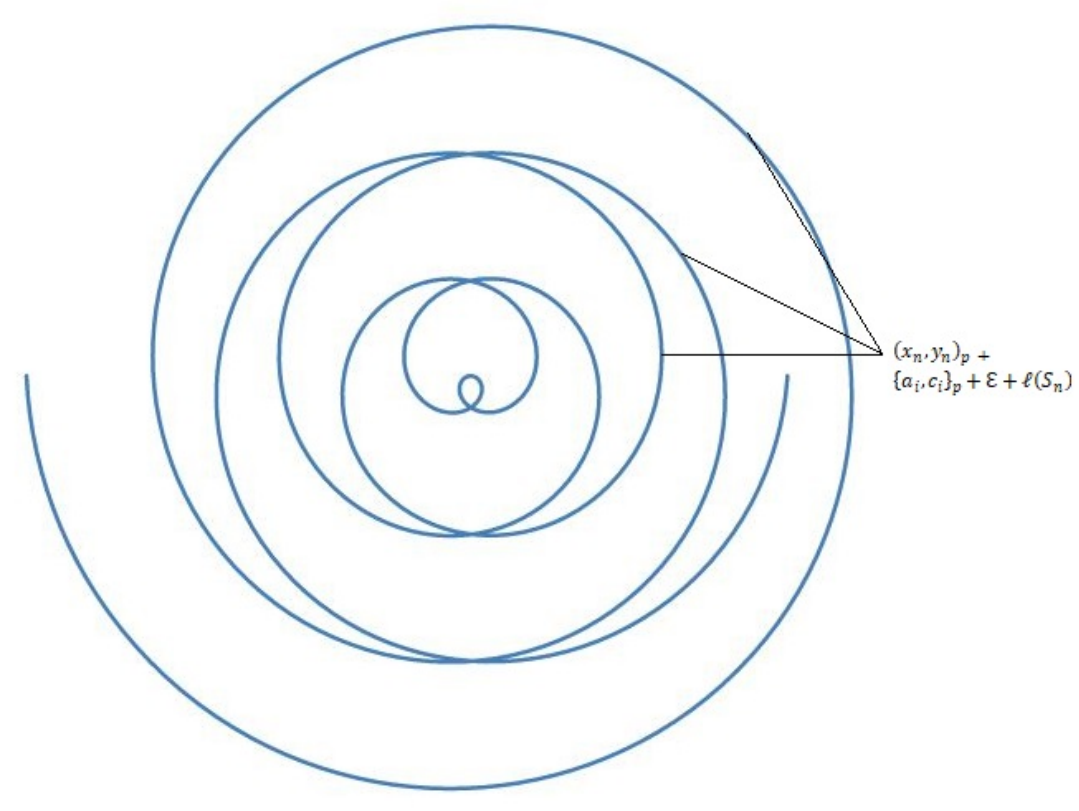

Figure (d) 


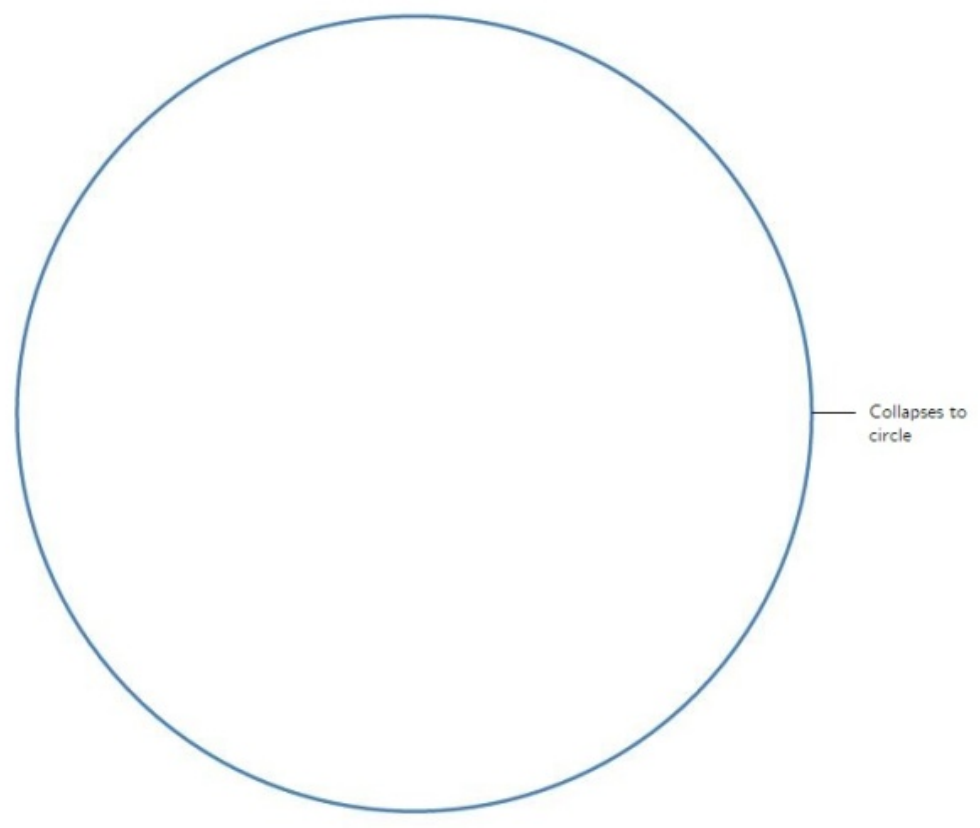

Figure (e)

An extensive analysis could be done regarding the properties of the 5 figures portrayed upwards. The equation governing the figures is $r=a+c+b \theta$ where the intervals of $a, b, c$ is $[-10,10]$ and $\theta$ is $0 \leq \theta \leq 12 \pi$. $b \theta$ is the parameter controlling the curves while $a$ amplifies $c$ and $c$ amplifies $a$. Figure (a) shows the normal Archimedes spiral with the pole being in the middle with the equation parameters being $a=0, c=0, b=$ -0.1 which is essentially the same if we plot the equation $r=a+b \theta$. The interesting property started to emerge from figure (b) which is an ellipse with one side being smashed to make a cone, which we prefer to say as the smashed ellipse more than an oval. The base points $\left(x_{0}, y_{0}\right)$ have been taken as a pole at the figure (a) which is the entropy function $S_{1}$. From that upon altering the values as $a=0, c=-4.7, b=1$ the pole emerge as a smashed ellipse which is revolving around the attractor boundary point $\partial_{\mathcal{A}}$ as we proceed further. The entropy function is now $S_{2}$ which is greater than $S_{1}$. Upon altering the parameters again as $a=-3.1, c=-4.8, b=1$ in figure (c), the smashed ellipse created another smashed ellipse around itself with a kink at its right side with the entropy function $S_{3}$, then upon altering the parameters as $a=-10, c=$ $0.9, b=-9.8$ the figure (d) emerges which is just repetitions of figure (c) with entropy function $S_{4}$. This will 
tend to continue but the whole process collapsed to a circle in figure (e) when we change the parameter as $a=-10, c=-10, b=0$. This gives us hint that any process can continue forever unless the angular parameter $b \theta$ has been turned to 0 . This proves that, there exists an equivalence closure and also exists a close union such that a repetition occurs in the form $S_{p}=S_{1} \sum_{i=2}^{\infty} S_{i}$ keeping $S$ as the entropy $\exists\left(S_{\infty}=\cdots S_{3}=S_{2}\right)>$ $S_{1}$ if we ignore the kink in the figure (b), as the smashed ellipse produces first after the kink produced which takes effect from figure (c), the equivalence closure can be given for all points $i$ in the whole set $X$ where $i^{\text {th }}$ points $\in I$ as;

$$
\coprod_{i \in I} X_{I} / \sim
$$

Where repetition with equal valued functions of entropy are seen taking place from the $S_{2}$ onwards such that $S_{i}$ are acting on the boundary domain $\partial \mathcal{A}_{p}$ where each smashed ellipse behaves as a pole while giving numerous iterations around the base points $\left(x_{0}, y_{0}\right)$. As the points that are travelling through this Hamiltonian trajectories are of fixed density throughout, its possible to incorporate the Liouville's theorem for this space, if and only if, we take, where the density given by $\rho$ takes on the functions of canonical coordinates $q_{i}$ and the conjugate momenta $p_{i}$, where $i=1 \ldots . n$, we will get a 3 -tuple relationship $(\rho, \rho \dot{q}, \rho \dot{p})$ with the Hamiltonian flow $H$ being conserved throughout the system, the equation reads as [4];

$$
\begin{aligned}
\frac{d \rho}{d t} & =\frac{\partial \rho}{\partial t}+\frac{\partial q}{\partial t} \frac{d \rho}{d q}+\frac{\partial p}{\partial t} \frac{d \rho}{d p} \\
& =\frac{\partial \rho}{\partial t}+\dot{q} \frac{\partial \rho}{\partial q}+\dot{p} \frac{\partial \rho}{\partial p} \\
& =\frac{\partial \rho}{\partial t}+\frac{\partial(\dot{q} \rho)}{\partial q}+\frac{\partial(\dot{q} \rho)}{\partial p}-\rho\left(\frac{\partial \dot{q}}{\partial q}+\frac{\partial \dot{p}}{\partial p}\right) \\
& =\frac{\partial \rho}{\partial t}+\frac{\partial(\dot{q} \rho)}{\partial q}+\frac{\partial(\dot{q} \rho)}{\partial p}-\rho\left(\frac{\partial}{\partial q}\left(\frac{\partial H}{\partial p}\right)+\frac{\partial}{\partial p}\left(-\frac{\partial H}{\partial q}\right)\right) \\
& \exists \rho\left(\frac{\partial}{\partial q}\left(\frac{\partial H}{\partial p}\right)+\frac{\partial}{\partial p}\left(-\frac{\partial H}{\partial q}\right)\right)=0
\end{aligned}
$$


The pole in the equation $r=a+c+b \theta$ which acts as the equation $r=a+b \theta$ at the base points $\left(x_{0}, y_{0}\right)$ can be stated providing $\theta_{1}=0, \theta_{2}=\theta$ can be expressed as;

$$
\frac{b}{2}\left[\theta \sqrt{1+\theta^{2}}+\operatorname{In}\left(\theta+\sqrt{1+\theta^{2}}\right)\right]
$$

While progressing $\theta_{1}=0$ got displayed in the -+ quadrant left taking the area generated through the poles by the smashed sphere as given by incomplete elliptic integral of the $2^{\text {nd }}$ kind $E(x, k)$ with the major radius being $a$ and minor radius being $b$ having the norm $a \geq b$ and $\frac{\pi}{2} \geq \theta \geq 0$ as expressed by the length $\ell$ of the closed curve [7];

$$
\begin{gathered}
a E\left(\frac{x\left(\theta_{0}\right)}{a}, k\right)-a E\left(\frac{x\left(\theta_{1}\right)}{a}, k\right) \\
x(\theta)=r(\theta) \cos \theta, k=\sqrt{1-\left(\frac{b}{a}\right)^{2}} \\
r(\theta)^{2}=\frac{a^{2} b^{2}}{b^{2} \cos ^{2} \theta+a^{2} \sin ^{2} \theta} \\
\theta_{0}=0^{\circ}, \theta_{1}=360^{\circ}, \theta=\left[0^{\circ} \rightarrow 90^{\circ}\right]
\end{gathered}
$$

This is the equation of the second pole with the entropy function $S_{2}$. The equation of the third generated pole with the kink is given by;

$$
\begin{gathered}
\left\{\begin{array}{c}
a E\left(\frac{x\left(\theta_{0}\right)}{a}, k\right)-a E\left(\frac{x\left(\theta_{1}\right)}{a}, k\right) \\
x(\theta)=r(\theta) \cos \theta, k=\sqrt{1-\left(\frac{b}{a}\right)^{2}} \\
-(\pi(b \times d)+2(b \times d))+2(\pi(b \times e)+2(b \times e)) \\
\theta_{0}=0^{\circ}, \theta_{1}=360^{\circ}, \theta=\left[0^{\circ} \rightarrow 90^{\circ}\right]
\end{array}\right. \\
\text { where }\left\{\begin{array}{c}
a^{2} b^{2} \\
d=\frac{m}{n} \\
e=\frac{p}{q} \\
n, q \neq 0 \\
q>n
\end{array}\right.
\end{gathered}
$$


Then this trajectory repeats itself along the boundary point $\partial \mathcal{A}_{p}$ with the relation $S_{p}=S_{1} \sum_{i=2}^{\infty} S_{i}$ keeping $S$ as the entropy $\exists\left(S_{\infty}=\cdots S_{3}=S_{2}\right)>S_{1}$ over dynamical iterations. To establish our relation of the violating thermodynamics, we can construct a set $P^{0}$ obeying the relation $P^{0}=\left\{x \in \mathcal{J}: \tau_{p}{ }^{k} x \notin \mathcal{J} \forall \mathcal{J}>0\right\}$ provided its important that we take $\forall \mathcal{J}>0$ and not for some $\mathcal{J}>0$ otherwise the relation won't suffice.

Thus in formal definition this can be rewritten as $[6]$;

$$
P^{0}=\mathcal{J} \cap\left(\bigcap_{k=1}^{\infty} \tau_{p}{ }^{k}(X \backslash \mathcal{J})\right)
$$

Therefore, from this construction of $P^{0}$ it is evident that $x \in P^{0}$ satisfying $\tau_{p} x, \tau_{p}{ }^{2} x, \tau_{p}{ }^{k} x \notin \mathcal{J}$ which implies that $x \notin \tau_{p}{ }^{-k} P^{0}$ so, $P^{0} \cap \tau_{p}{ }^{-k} P^{0}=\phi$ which makes us to see that each set $\tau_{p}{ }^{-1} P^{0}, \tau_{p}{ }^{-2} P^{0}, \ldots \tau_{p}{ }^{-k} P^{0}$ is a pairwise disjoint. By properties of dynamical transformations, for each $k_{1}, k_{2} \in \mathbb{N}, k_{1}>k_{2}$, the relation becomes [5];

$$
\tau_{p}^{-k_{1} P^{0}} \cap \tau_{p}{ }^{-k_{2}} P^{0}=\tau_{p}{ }^{-k_{1}}\left(P^{0} \cap \tau_{p}{ }^{-\left(k_{1}-k_{2}\right)} P^{0}\right)=\phi
$$

Then, the inequality can only be disjoint in each set $P^{0}, \tau_{p}{ }^{-1} P^{0}, \tau_{p}{ }^{-2} P^{0}, \ldots \tau_{p}{ }^{-k} P^{0}$ are disjoint allowing the Haar measure to be invariant under $\mu(\mathcal{J})=0$ on the measure space $(\mathcal{J}, \Sigma)$ following [8];

$$
1 \geq \mu\left(\bigcup_{k=0}^{\infty} P^{0}\right)=\sum_{k=0}^{\infty} \mu\left(\tau_{p}{ }^{-k} P^{0}\right)=\sum_{k=0}^{\infty} \mu\left(P^{0}\right)
$$

Thus we can conclude that every repeating pole other than the entropy function $S_{1}$ generated by the generators $\left(x_{0}, y_{0}\right)$ around the domain of attractor boundary $\partial_{\mathcal{A}_{p}}$ is a equivalence closure with $\left(X_{I}\right)_{i \in I}$ having the indexed family of base points $\left(p_{i}\right)_{i \in I,\left(x_{0}, y_{0}\right) \in i}$ the relation suffices as [9];

$$
\bigvee_{i \in I} X_{I}=\coprod_{i \in I} X_{I} / \sim
$$

Which is closed through equivalence with the infinite iteration state occurring but the $S_{i-1}$ generates the states with the entropy being conserved thus violating the second law of thermodynamics according to the second law of thermodynamics, entropy of a closed system always tends to increase with time. [10]. 
$a+c+b \theta$ can be generalized to any number of variables as to $a+c+d+e+f+g \ldots+b \theta \forall a=c=$ $d=e=f=g \exists a_{1}=a, a_{2}=c, a_{3}=d, a_{4}=e, a_{5}=f, a_{6}=g \ldots a_{i}=n$, the equation becomes;

$$
\sum_{i=1}^{\infty} a_{i}+b \theta=r
$$

Let $b \theta_{\epsilon}, b \theta_{\epsilon+1}, b \theta_{\epsilon+2}, b \theta_{\epsilon+3}, b \theta_{\epsilon+4} \ldots b \theta_{\epsilon+n}$ be the set of points belong to $b \theta, \exists \sum b \theta_{\epsilon+n} \in b \theta$, then as $b \theta$ amplifies $r$, the stated equation becomes;

$$
\prod_{n=1}^{\infty} b \theta_{\epsilon-1+n}=r-\sum_{i=1}^{\infty} a_{i}
$$

Now, let $a_{\epsilon}, a_{\epsilon+1}, a_{\epsilon+2}, a_{\epsilon+3}$ be the element of $\sum a_{\epsilon+n}$. Then $c_{\epsilon}, c_{\epsilon+1}, c_{\epsilon+2}, c_{\epsilon+3}$ be the element of $\sum c_{\epsilon+n}$ such that the relation suffices into an equality;

$$
\begin{array}{cccccc}
a_{\epsilon} & = & c_{\epsilon} & & d_{\epsilon} & \cdots \\
a_{\epsilon+1} & = & c_{\epsilon+1} & = & d_{\epsilon+1} & \cdots \\
a_{\epsilon+2} & = & c_{\epsilon+2} & = & d_{\epsilon+2} & \cdots \\
\vdots & = & \vdots & & \vdots & \cdots \\
a_{\epsilon+n} & = & c_{\epsilon+n} & = & d_{\epsilon+n} & \cdots
\end{array}
$$

Then, $\sum_{n=1}^{\infty} a_{\epsilon-1+n}$ amplifies $\sum_{n=1}^{\infty} c_{\epsilon-1+n} \exists F(z)$ on a subset $\{a, c\}$ of a open set $\{a, c, d, e \ldots\}$ where the point $p$ on $\{a, c\}$ as $\{a, c\}_{p}$ revolves through $r$ via $F(z)+2 \pi r$ which being a continuous function on $\{a, c\}_{p}$ $\forall\left\{a_{1}, c_{1}\right\}_{p}$ makes a turn on the open neighborhood of $\left\{a_{2}, c_{2}\right\}_{p}$ in a way as;

$$
\begin{gathered}
\left\{a_{1}, c_{1}\right\}_{p}+\left\{\begin{array}{c}
a E\left(\frac{x\left(\theta_{0}\right)}{a}, k\right)-a E\left(\frac{x\left(\theta_{1}\right)}{a}, k\right) \\
x(\theta)=r(\theta) \cos \theta, k=\sqrt{1-\left(\frac{b}{a}\right)^{2}} \\
r(\theta)^{2}=\frac{a^{2} b^{2}}{b^{2} \cos ^{2} \theta+a^{2} \sin ^{2} \theta} \\
\theta_{0}=0^{\circ}, \theta_{1}=360^{\circ}, \theta=\left[0^{\circ} \rightarrow 90^{\circ}\right]
\end{array}\right. \\
\text { where }\left\{\begin{array}{l}
d=\frac{m}{n} \\
e=\frac{p}{q} \\
n, q \neq 0 \\
q>n
\end{array}\right.
\end{gathered}
$$


Rotates the subset of base points denoted by the function $F(z)$ as $\left(x_{0}, y_{0}\right)_{p} \stackrel{\text { Base }}{\longrightarrow}\left(x_{1}, y_{1}\right)_{p} \rightarrow\left(x_{2}, y_{2}\right)_{p} \rightarrow$ $\left(x_{n}, y_{n}\right)_{p} \exists n \in \mathbb{R}, n \geq 0$ the monodromy occurs in $\partial \mathcal{A}_{p}$ surrounding $\left(x_{0}, y_{0}\right) \exists$ the initial point makes $2 \pi r$ revolutions around the $\left(x_{0}, y_{0}\right)_{p} \forall\left\{a_{n}, c_{n}\right\}_{p}$ while transforming through the entropy function $S_{1} \sum_{n=2}^{\infty} S_{n}$, provided we denote the parameter;

$$
-(\pi(b \times d)+2(b \times d))+2(\pi(b \times e)+2(b \times e))=\ell\left(\sum_{n=2}^{\infty} S_{n}\right)
$$

And,

$$
\left\{\begin{array}{c}
a E\left(\frac{x\left(\theta_{0}\right)}{a}, k\right)-a E\left(\frac{x\left(\theta_{1}\right)}{a}, k\right) \\
x(\theta)=r(\theta) \cos \theta, k=\sqrt{1-\left(\frac{b}{a}\right)^{2}} \\
r(\theta)^{2}=\frac{a^{2} b^{2}}{b^{2} \cos ^{2} \theta+a^{2} \sin ^{2} \theta} \\
\theta_{0}=0^{\circ}, \theta_{1}=360^{\circ}, \theta=\left[0^{\circ} \rightarrow 90^{\circ}\right]
\end{array} \mid\right.
$$

As the starting after the base point, the equation stands out as;

$$
\begin{array}{ccc}
\left(x_{0}, y_{0}\right)_{p} & + & \left\{a_{1}, c_{1}\right\}_{p}+\mathcal{E}+\ell\left(S_{2}\right) \\
\left(x_{1}, y_{1}\right)_{p} & + & \left\{a_{2}, c_{2}\right\}_{p}+\mathcal{E}+\ell\left(S_{3}\right) \\
\left(x_{2}, y_{2}\right)_{p} & + & \left\{a_{3}, c_{3}\right\}_{p}+\mathcal{E}+\ell\left(S_{4}\right) \\
\vdots & + & \vdots \\
\left(x_{n}, y_{n}\right)_{p} & + & \left\{a_{i}, c_{i}\right\}_{p}+\mathcal{E}+\ell\left(S_{n}\right)
\end{array}
$$

Provided, $S_{2}=S_{3}=S_{4}=\cdots=S_{n} \forall\left(x_{0}, y_{0}\right)$ there exists the relation;

$$
p \geq 0\left(\bigcap_{\left\{a_{i}, c_{i}\right\} \in \partial_{A p}} x_{n}, y_{n}\right)
$$

Where,

$$
\sum_{i=1}^{\infty} a_{i}, c_{i} \underset{\text { acts on }}{\longrightarrow} \sum_{n=0}^{\infty} x_{n}, y_{n}
$$

Given, the relations, it is evident from $\left(x_{0}, y_{0}\right)_{p}+\left\{a_{1}, c_{1}\right\}_{p}+\varepsilon+\ell\left(S_{2}\right)$ that the norm $\left\{a_{1}, c_{1}\right\}_{p}+\mathcal{E}+\ell\left(S_{2}\right)$ revolves around $\left(x_{0}, y_{0}\right)_{p}$, likewise, in $\left(x_{1}, y_{1}\right)_{p}+\left\{a_{2}, c_{2}\right\}_{p}+\mathcal{E}+\ell\left(S_{3}\right)$, the norm $\left\{a_{2}, c_{2}\right\}_{p}+\mathcal{E}+\ell\left(S_{3}\right)$ revolves around $\left(x_{1}, y_{1}\right)_{p}$, therefore, with the inclusion on the base points as $+\left(x_{0}, y_{0}\right)_{p}$ and the points above the base as $-\left(x_{1}, y_{1}\right)_{p},-\left(x_{2}, y_{2}\right)_{p}$ up to $-\left(x_{n}, y_{n}\right)_{p}$, it has been evident that taking these relations as the 
diagonal component we would get a metric index as two types, the spinner and the spreader. The spinner is the spinor provided we establish a relation within a bounded limit as to say the equation $a+c+b \theta$ with interval $a:=[-10,10], b:=[-10,10], c:=[-10,10]$ keeping the angular parameter $b \theta>0$ and $b \theta<0$ we get the spinor $\gamma_{j}=\left(\begin{array}{l}\Psi^{+} \\ \Psi^{-}\end{array}\right)$where $\Psi^{+}$is the anti-clockwise and $\Psi^{-}$is the clockwise, there is devoid of collapsing the base points $\left(x_{0}, y_{0}\right)$ into a 'core singularity' however, if we take the spreader relation as $\tilde{\gamma}_{j}=\left(\begin{array}{c}\Phi^{+} \\ \Phi^{-}\end{array}\right)$making the parameter $b \theta=0$ while keeping the other intervals fixed, $\Phi^{+}$suffices for contraction of the circle and $\Phi^{-}$suffices for expansion of the circle, we would expect the 'core singularity' to act on the metric $\zeta_{\mu \nu} d \eta^{\mu} d \eta^{\nu}$ with the signature $(+,-,-,-, \ldots,-)$ represented as with the core singularity point $\Phi^{+}$in the form $\zeta_{\mu \nu}{ }^{\Phi^{+}}$, given the metric $\zeta_{\mu \nu}$ as ;

$$
\left(\begin{array}{ccc}
\left(x_{0}, y_{0}\right)_{p}+\left\{a_{1}, c_{1}\right\}_{p}+\mathcal{E}+\ell\left(S_{2}\right) & 0 & 0 \\
\vdots & \ddots & \vdots \\
0 & 0 & -\left(\left(x_{n}, y_{n}\right)_{p}+\left\{a_{i}, c_{i}\right\}_{p}+\mathcal{E}+\ell\left(S_{n}\right)\right)
\end{array}\right)
$$

Provided we are getting three kernels to map the vector field in the singularity as such;

- $\operatorname{ker}\left(\zeta_{\mu \nu}{ }^{\Psi^{+}} \rightarrow \zeta_{\mu \nu}{ }^{\Phi^{+}}\right)$

- $\operatorname{ker}\left(\zeta_{\mu \nu}{ }^{\Psi^{-}} \rightarrow \zeta_{\mu \nu}{ }^{\Phi^{+}}\right)$

- $\operatorname{ker}\left(\zeta_{\mu \nu}{ }^{\Phi^{-}} \rightarrow \zeta_{\mu \nu}{ }^{\Phi^{+}}\right)$

And the ran function runs through 4 identities to satisfy as below in order;
- $\overline{\operatorname{ran}\left(\zeta_{\mu \nu}{ }^{\Psi^{+}} \rightarrow \zeta_{\mu \nu}{ }^{\Psi^{-}}\right)}$
- $\overline{\operatorname{ran}\left(\zeta_{\mu \nu}{ }^{\Psi^{-}} \rightarrow \zeta_{\mu \nu}{ }^{\Psi^{+}}\right)}$
- $\overline{\operatorname{ran}\left(\zeta_{\mu \nu}{ }^{\Psi^{+}} \rightarrow \zeta_{\mu \nu}{ }^{\Phi^{-}}\right)}$
- $\overline{\operatorname{ran}\left(\zeta_{\mu \nu}{ }^{\Psi^{-}} \rightarrow \zeta_{\mu \nu}{ }^{\Phi^{-}}\right)}$ 
The ker part is invariant taking all the partial sums of $\ell\left(\sum_{n=2}^{\infty} S_{n}\right)$ while the latter part derives the telescoping series for a relation as $\tau_{p}$ is a measure preserving endomorphism of $\mathcal{J}$ on the operator space $\zeta_{\mu \nu}$ as noted by the relations;

$$
\zeta_{\mu \nu}\left(\xi\left(x_{0}, y_{0}\right)\right)=\xi\left(T\left(\sum_{n=1}^{\infty} x_{n}, y_{n}\right)\right)
$$

Which satisfies the limit of the series as given below taking all the four $\overline{r a n}$ relations;

$$
\begin{aligned}
& \lim _{N \rightarrow \infty} \frac{1}{N} \sum_{n=0}^{N-1}\left(\zeta_{\mu \nu}\right)^{n}\left(\zeta_{\mu \nu}{ }^{\Psi^{+}}-\zeta_{\mu \nu}{ }^{\Psi^{-}}\right)=\lim _{N \rightarrow \infty} \frac{1}{N}\left(\zeta_{\mu \nu}{ }^{\Psi^{+}}-\left(\zeta_{\mu \nu}{ }^{\Psi^{-}}\right)^{N}\right) \\
& \lim _{N \rightarrow \infty} \frac{1}{N} \sum_{n=0}^{N-1}\left(\zeta_{\mu \nu}\right)^{n}\left(\zeta_{\mu \nu}{ }^{\Psi^{-}}-\zeta_{\mu \nu}{ }^{\Psi^{+}}\right)=\lim _{N \rightarrow \infty} \frac{1}{N}\left(\zeta_{\mu \nu}{ }^{\Psi^{-}}-\left(\zeta_{\mu \nu}{ }^{\Psi^{+}}\right)^{N}\right) \\
& \lim _{N \rightarrow \infty} \frac{1}{N} \sum_{n=0}^{N-1}\left(\zeta_{\mu \nu}\right)^{n}\left(\zeta_{\mu \nu}{ }^{\Psi^{+}}-\zeta_{\mu \nu}{ }^{\Phi^{-}}\right)=\lim _{N \rightarrow \infty} \frac{1}{N}\left(\zeta_{\mu \nu}{ }^{\Psi^{+}}-\left(\zeta_{\mu \nu}{ }^{\Phi^{-}}\right)^{N}\right) \\
& \lim _{N \rightarrow \infty} \frac{1}{N} \sum_{n=0}^{N-1}\left(\zeta_{\mu \nu}\right)^{n}\left(\zeta_{\mu \nu}{ }^{\Psi^{-}}-\zeta_{\mu \nu}{ }^{\Phi^{-}}\right)=\lim _{N \rightarrow \infty} \frac{1}{N}\left(\zeta_{\mu \nu}{ }^{\Psi^{-}}-\left(\zeta_{\mu \nu}{ }^{\Phi^{-}}\right)^{N}\right)
\end{aligned}
$$

Therefore, in the measure space, $(\mathcal{A}, \sim, \mathcal{J}, \Sigma, \mu)$, provided $\mu(\mathcal{J})$ is finite and nonzero, the sojourn time [11] can be defined as the time spent on the set $\delta$ having the Haar measuring function $\mu(\delta)$ defined as,

$$
\frac{\mu(\delta)}{\mu(\mathcal{J})}=\frac{1}{\mu(\mathcal{J})} \int \varkappa \delta d \mu=\lim _{n \rightarrow \infty} \frac{1}{n} \sum_{k=0}^{n-1} \varkappa \delta\left(\tau_{p}{ }^{k} \mathcal{J}\right)
$$

Where $\varkappa \delta$ is the indicator function of set $\delta$ with two elements $\{0,1\} \exists \delta \rightarrow 1$ is probable in the measuring space $(\mathcal{A}, \sim, \mathcal{J}, \Sigma, \mu)$ which is the number of iterations over $M_{\epsilon}$ provided for the initial point $k_{0} \cong$ $\left(x_{0}, y_{0}\right)=0 \exists M_{\epsilon}=k_{i}-k_{i-1}$ given as;

$$
\frac{M_{1}+M_{2}+\cdots+M_{\epsilon}}{\epsilon}=\frac{\mu(\mathcal{J})}{\mu(\delta)}
$$

While the ergodicity converges to $\tau_{p}=\Phi^{+} \cong \infty$ upon the metric space $\zeta_{\mu \nu}$ in time $t$ expressed as; 


$$
\frac{1}{\tau_{p}} \int_{0}^{\tau_{p}}\left(\zeta_{\mu \nu}\right)_{t} d t
$$

The singularity being satisfied as $\left(x_{0}, y_{0}\right)^{\Phi^{+}}$the constant entropy is preserved without any singularity as;

$$
\frac{\left(x_{n}, y_{n}\right)}{\left(x_{0}, y_{0}\right)^{\Phi^{+}}} \approx\left(a_{i-1}, b_{i-1}\right) \exists i \neq 1,1<i \leq \infty
$$

Therefore, the relation between the spinor $\gamma_{j}$ and the spreader $\tilde{\gamma}_{j}$ can be defined as a matrix product;

$$
\left(\begin{array}{l}
\Psi^{+} \\
\Psi^{-}
\end{array}\right) \times\left(\begin{array}{l}
\Phi^{+} \\
\Phi^{-}
\end{array}\right)=\left[\begin{array}{ll}
\Psi^{+} \Phi^{+} & \Psi^{+} \Phi^{-} \\
\Psi^{-} \Phi^{+} & \Psi^{-} \Phi^{-}
\end{array}\right]=f_{i j}
$$

The determinant if can be computed of the matrix $f_{i j}$ then this yields to the result;

$$
\operatorname{Det}\left(f_{i j}\right)=\Psi^{+} \Phi^{+} \Psi^{-} \Phi^{-}-\Psi^{-} \Phi^{+} \Psi^{+} \Phi^{-}=0
$$

This satisfies the inclusion of the base points at the starting of the ergodicity through a deterministic curve of repeated iterations as the corresponding base point equations to be developed in the way by inclusion of $\operatorname{Det}\left(f_{i j}\right)$ into $\left(x_{0}, y_{0}\right)_{p}$ as;

$$
\left(x_{D e t}\left[\begin{array}{ll}
\Psi^{+} \Phi^{+} & \Psi^{+} \Phi^{-} \\
\Psi^{-} \Phi^{+} & \Psi^{-} \Phi^{-}
\end{array}\right], y_{D e t}\left[\begin{array}{ll}
\Psi^{+} \Phi^{+} & \Psi^{+} \Phi^{-} \\
\Psi^{-} \Phi^{+} & \Psi^{-} \Phi^{-}
\end{array}\right)_{p}\right.
$$

Satisfying the relation $\left(a_{i-1}, b_{i-1}\right) \exists i=1$ as the constant time element to be preserved in the metric with the values taking place beyond $\left(a_{i-1}, b_{i-1}\right) \exists i=1$ in $\left(a_{i-1}, b_{i-1}\right) \exists i \neq 1,1<i \leq \infty$ with the solutions being of the order;

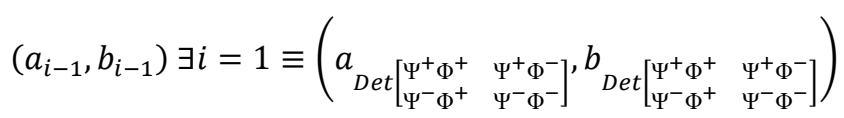

Note that, as this is an element of constant time, so, $\left(\left(a_{i-1}, b_{i-1}\right) \exists i=1\right) \notin\left(\left(a_{i-1}, b_{i-1}\right) \exists i \neq 1,1<i \leq \infty\right)$ as considered in spatial iterations but remains there if we replace the identity $i$ as $d$ in case of $\left(a_{i-1}, b_{i-1}\right) \exists i=1$ such that it becomes, $\left(a_{d-1}, b_{d-1}\right) \exists d=1$ and including this into $\left(a_{i-1}, b_{i-1}\right) \exists i \neq 1,1<$ $i \leq \infty$ as;

$$
\left(a_{(d-1)+(i-1)}, b_{(d-1)+(i-1)}\right) \exists i \neq 1, d=1,1<i \leq \infty
$$

The metric being modified keeping all the previous values as the same being represented as; 
$\left(\begin{array}{ccc}\left(x_{\operatorname{Det}\left(f_{i j}\right)}, y_{\operatorname{Det}\left(f_{i j}\right)}\right)_{p}+\left\{a_{\operatorname{Det}\left(f_{i j}\right)+1}, c_{\operatorname{Det}\left(f_{i j}\right)+1}\right\}_{p}+\varepsilon+\ell\left(S_{2}\right) & 0 & 0 \\ \vdots & \ddots & \vdots \\ 0 & 0 & -\left(\left(x_{\operatorname{Det}\left(f_{i j}\right)+n}, y_{\operatorname{Det}\left(f_{i j}\right)+n}\right)_{p}+\left\{a_{\operatorname{Det}\left(f_{i j}\right)+i}, c_{\operatorname{Det}\left(f_{i j}\right)+i}\right\}_{p}+\varepsilon+\ell\left(S_{n}\right)\right)\end{array}\right)$

It is evident from the fact that $\left(x_{0}, y_{0}\right)$ are base points with the entropy function $S_{1}$ and $\left\{a_{1}, c_{1}\right\}_{p}+\varepsilon+\ell\left(S_{2}\right)$ revolves around $\left(x_{0}, y_{0}\right)_{p}$ to give the entropy function of the associated curve $\ell\left(S_{2}\right)$ which continues up to $\left\{a_{i}, c_{i}\right\}_{p}+\varepsilon+\ell\left(S_{n}\right)$ revolving around $\left(x_{n}, y_{n}\right)_{p}$ to give the final state although the final state is itself an iteration of a more sustained final state obeying the entropy formulation $S_{p}=S_{1} \sum_{i=2}^{\infty} S_{i}$ keeping $S$ as the entropy $\exists\left(S_{\infty}=\cdots S_{3}=S_{2}\right)>S_{1}$. Therefore, there rises the question that if $\sum_{i=2}^{\infty} S_{i}$ is constant on the grounds of the equivalence

closure $\bigvee_{i \in I} X_{I}=\bigsqcup_{i \in I} X_{I} / \sim$ then there arises a conditional entropy which describes the outcome of the points $\sum_{n=1}^{\infty} S_{n}$ provided we know the initial base points revolving around the boundary $\partial \mathcal{A}_{\left(x_{0}, y_{0}\right) p}$ which has an outcome value on $\sum_{n=1}^{\infty}\left(x_{n}, y_{n}\right)_{p}$ such that, it satisfies the relation [12];

$$
\begin{aligned}
& \mathscr{H}\left(\sum_{n=1}^{\infty}\left(x_{n}, y_{n}\right)_{p} \mid\left(x_{0}, y_{0}\right)_{p}\right) \\
& =-\sum_{\frac{\sum_{\overline{\sum_{n=1}}\left(x_{n}, y_{n}\right)_{p} \in \ell}^{\infty} \in \overline{\ell\left(S_{1}\right)}}{\widetilde{\sum_{n=2}^{\infty}} s_{n}}} P\left(\left(x_{0}, y_{0}\right)_{p}, \sum_{n=1}^{\infty}\left(x_{n}, y_{n}\right)_{p}\right) \log \frac{P\left(\left(x_{0}, y_{0}\right), \sum_{n=1}^{\infty}\left(x_{n}, y_{n}\right)_{p}\right)}{P\left(\sum_{n=1}^{\infty}\left(x_{n}, y_{n}\right)_{p}\right)}
\end{aligned}
$$

Where $\widetilde{\ell\left(S_{1}\right)}$ is the support set of $\left(x_{0}, y_{0}\right) p$ and $\ell \widetilde{\sum_{n=2}^{\infty}} S_{n}$ is the support set of $\sum_{n=1}^{\infty}\left(x_{n}, y_{n}\right)_{p}$. Note, that the definition entails the relation;

$$
\mathscr{H}\left(\sum_{n=1}^{\infty}\left(x_{n}, y_{n}\right)_{p} \mid\left(x_{0}, y_{0}\right)_{p}\right)=\mathbb{E}\left(f\left(\left(x_{0}, y_{0}\right)_{p}, \sum_{n=1}^{\infty}\left(x_{n}, y_{n}\right)_{p}\right)\right)
$$

Where,

$$
f:\left(\overline{\left(x_{0}, y_{0}\right) p}, \overline{\left.\sum_{n=1}^{\infty}\left(x_{n}, y_{n}\right)_{p}\right)}\right) \rightarrow-\log \left(P\left(\sum_{n=1}^{\infty}\left(x_{n}, y_{n}\right)_{p} \mid\left(x_{0}, y_{0}\right)_{p}\right)\right)
$$

In which $f$ associates to $\left(f\left(\sum_{n=1}^{\infty}\left(x_{n}, y_{n}\right)_{p},\left(x_{0}, y_{0}\right)_{p}\right)\right)$ the information content which will be the amount of information needed to describe the properties of $\left(\sum_{n=1}^{\infty}\left(x_{n}, y_{n}\right)_{p}=\left(\overline{\sum_{n=1}^{\infty}\left(x_{n}, y_{n}\right)_{p}}\right),\left(x_{0}, y_{0}\right)_{p}=\overline{\left(x_{0}, y_{0}\right)_{p}}\right)$ for a large number of iterations $f\left(\sum_{n=1}^{\infty}\left(x_{n}, y_{n}\right)_{p},\left(x_{0}, y_{0}\right)_{p}\right)$. 
We have denoted the support sets of $\overline{\sum_{n=1}^{\infty}\left(x_{n}, y_{n}\right)_{p}}$ being $\ell \widetilde{\sum_{n=2}^{\infty}} S_{n}$ and $\overline{\left(x_{0}, y_{0}\right) p}$ being $\overline{\ell\left(S_{1}\right)}$ where $\sum_{n=1}^{\infty}\left(x_{n}, y_{n}\right)_{p}$ have the probability mass function $P_{\sum_{n=1}^{\infty}\left(x_{n}, y_{n}\right)_{p}}\left(\overline{\sum_{n=1}^{\infty}\left(x_{n}, y_{n}\right)_{p}}\right)$ where unconditional entropy of $\sum_{n=1}^{\infty}\left(x_{n}, y_{n}\right)_{p}$ has been calculated as $\mathscr{H}\left(\sum_{n=1}^{\infty}\left(x_{n}, y_{n}\right)_{p}\right):=\mathbb{E}\left[I\left(\sum_{n=1}^{\infty}\left(x_{n}, y_{n}\right)_{p}\right)\right]$, that is,

$$
\begin{aligned}
& \mathscr{H}\left(\sum_{n=1}^{\infty}\left(x_{n}, y_{n}\right)_{p}\right)
\end{aligned}
$$

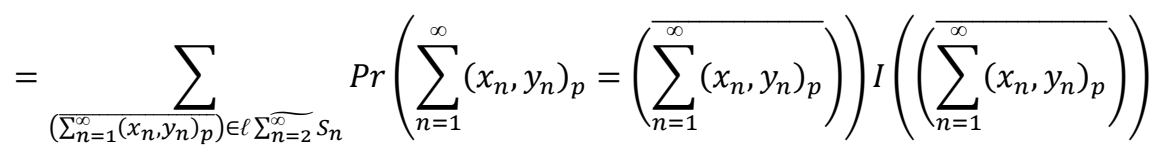

$$
\begin{aligned}
& =-\sum_{\left(\overline{\left.\sum_{n=1}^{\infty}\left(x_{n}, y_{n}\right)_{p}\right) \in \ell \sum_{n=2}^{\infty} S_{n}}\right.} P_{\sum_{n=1}^{\infty}\left(x_{n}, y_{n}\right)_{p}}\left(\overline{\sum_{n=1}^{\infty}\left(x_{n}, y_{n}\right)_{p}}\right) \log _{2} P_{\sum_{n=1}^{\infty}\left(x_{n}, y_{n}\right)_{p}}\left(\overline{\left.\sum_{n=1}^{\infty}\left(x_{n}, y_{n}\right)_{p}\right)}\right.
\end{aligned}
$$

Where $I\left(\left(\overline{\sum_{n=1}^{\infty}\left(x_{n}, y_{n}\right)_{p}}\right)\right)_{i}$ is the information content that entails the outcome of $\sum_{n=1}^{\infty}\left(x_{n}, y_{n}\right)_{p}$ taking the value $\left(\left(\overline{\sum_{n=1}^{\infty}\left(x_{n}, y_{n}\right)_{p}}\right)\right)_{i}$. Therefore, the conditional expectation of the associated entropy can be defined as the entropy of $\sum_{n=1}^{\infty}\left(x_{n}, y_{n}\right)_{p}$ with the known value of $\left(x_{0}, y_{0}\right)_{p}$ having the expectation value $[13,14]$;

$$
\begin{aligned}
& \mathscr{H}\left(\sum_{n=1}^{\infty}\left(x_{n}, y_{n}\right)_{p} \mid\left(x_{0}, y_{0}\right)_{p}=\overline{\left(x_{0}, y_{0}\right)_{p}}\right) \\
& =-\sum_{\overline{\sum_{n=1}^{\infty}\left(x_{n}, y_{n}\right)_{p} \in \ell \sum_{n=2}^{\infty} s_{n}}} \operatorname{Pr} \sum_{n=1}^{\infty}\left(x_{n}, y_{n}\right)_{p}=\overline{\sum_{n=1}^{\infty}\left(x_{n}, y_{n}\right)_{p}} \mid\left(x_{0}, y_{0}\right)_{p} \\
& =\overline{\left(x_{0}, y_{0}\right)_{p}} \log _{2} \operatorname{Pr} \sum_{n=1}^{\infty}\left(x_{n}, y_{n}\right)_{p}=\overline{\sum_{n=1}^{\infty}\left(x_{n}, y_{n}\right)_{p}} \mid\left(x_{0}, y_{0}\right)_{p}
\end{aligned}
$$

Now, it is to be noted that in case of $\mathscr{H}\left(\sum_{n=1}^{\infty}\left(x_{n}, y_{n}\right)_{p} \mid\left(x_{0}, y_{0}\right)_{p}\right)$ the resultant averaging $\mathscr{H}\left(\sum_{n=1}^{\infty}\left(x_{n}, y_{n}\right)_{p} \mid\left(x_{0}, y_{0}\right)_{p}=\overline{\left(x_{0}, y_{0}\right)_{p}}\right)$ over every possible values of $\overline{\left(x_{0}, y_{0}\right)_{p}}$ that $\left(x_{0}, y_{0}\right)_{p}$ can also take provided the sum is taken over the convention $\sum_{n=1}^{\infty}\left(x_{n}, y_{n}\right)_{p}$ the expected value becomes, 
$E_{\left(x_{0}, y_{0}\right)_{p}}\left[\mathscr{H}\left(\sum_{n=1}^{\infty}\left(x_{n}, y_{n}\right)_{p} \mid\left(x_{0}, y_{0}\right)_{p}=\overline{\left(x_{0}, y_{0}\right)_{p}}\right)\right]$ for each and every possible values of $\left(x_{0}, y_{0}\right)_{p}$ with the probabilistic weight $P\left(\left(x_{0}, y_{0}\right)_{p}\right)$ suffices to the following;

$$
\begin{aligned}
& \mathscr{H}\left(\sum_{n=1}^{\infty}\left(x_{n}, y_{n}\right)_{p} \mid\left(x_{0}, y_{0}\right)_{p}\right) \equiv \sum_{\left(x_{0}, y_{0}\right)_{p} \in \overline{\ell\left(S_{1}\right)}} P\left(\left(x_{0}, y_{0}\right)_{p}\right) \mathscr{H}\left(\sum_{n=1}^{\infty}\left(x_{n}, y_{n}\right)_{p} \mid\left(x_{0}, y_{0}\right)_{p}=\overline{\left(x_{0}, y_{0}\right)_{p}}\right) \\
& =-\sum_{\left(x_{0}, y_{0}\right)_{p} \in \overline{\ell\left(S_{1}\right)}} P\left(\left(x_{0}, y_{0}\right)_{p}\right) \sum_{\sum_{n=1}^{\infty}\left(x_{n}, y_{n}\right)_{p} \in \ell \widetilde{\sum_{n=2}^{\infty}} S_{n}} P\left(\overline{\sum_{n=1}^{\infty}\left(x_{n}, y_{n}\right)_{p}} \mid \overline{\left(x_{0}, y_{0}\right)_{p}}\right) \log P\left(\overline{\sum_{n=1}^{\infty}\left(x_{n}, y_{n}\right)_{p}} \mid \overline{\left(x_{0}, y_{0}\right)_{p}}\right) \\
& =-\sum_{\left(x_{0}, y_{0}\right)_{p} \in \overline{\ell\left(S_{1}\right)}} \sum_{\sum_{n=1}^{\infty}\left(x_{n}, y_{n}\right)_{p} \in \ell \overline{\sum_{n=2}^{\infty}} s_{n}} P\left(\overline{\left(x_{0}, y_{0}\right)_{p}}, \overline{\sum_{n=1}^{\infty}\left(x_{n}, y_{n}\right)_{p}}\right) \log P\left(\overline{\sum_{n=1}^{\infty}\left(x_{n}, y_{n}\right)_{p}} \mid \overline{\left(x_{0}, y_{0}\right)_{p}}\right) \\
& =-\sum_{\left(x_{0}, y_{0}\right)_{p} \in \overline{\ell\left(S_{1}\right)}} P\left(\overline{\left(x_{0}, y_{0}\right)_{p}}, \overline{\sum_{n=1}^{\infty}\left(x_{n}, y_{n}\right)_{p}}\right) \log \frac{P\left(\overline{\left(x_{0}, y_{0}\right)_{p}}, \overline{\sum_{n=1}^{\infty}\left(x_{n}, y_{n}\right)_{p}}\right)}{P\left(\overline{\left(x_{0}, y_{0}\right)_{p}}\right)} \\
& \sum_{n=1}^{\infty}\left(x_{n}, y_{n}\right)_{p} \in \ell \overline{\sum_{n=2}^{\infty}} S_{n} \\
& =\quad \sum_{\left(x_{0}, y_{0}\right)_{p} \in \overline{\ell\left(S_{1}\right)}} P\left(\overline{\left(x_{0}, y_{0}\right)_{p}}, \overline{\sum_{n=1}^{\infty}\left(x_{n}, y_{n}\right)_{p}}\right) \log \frac{P\left(\overline{\left(x_{0}, y_{0}\right)_{p}}\right)}{P\left(\overline{\left(x_{0}, y_{0}\right)_{p}}, \overline{\sum_{n=1}^{\infty}\left(x_{n}, y_{n}\right)_{p}}\right)} \\
& \sum_{n=1}^{\infty}\left(x_{n}, y_{n}\right)_{p} \in \ell \widetilde{\sum_{n=2}^{\infty}} S_{n} \\
& =0 \text { if }\left[S_{2}=S_{3}=\cdots=S_{n}\right]
\end{aligned}
$$

If the whole system can be defined by the random variables, $\left(x_{0}, y_{0}\right)_{p}$ and $\sum_{n=1}^{\infty}\left(x_{n}, y_{n}\right)_{p}$ then the joint entropy be determined as;

$$
\begin{aligned}
& \mathscr{H}\left(\left(x_{0}, y_{0}\right)_{p}, \sum_{n=1}^{\infty}\left(x_{n}, y_{n}\right)_{p}\right) \\
& =-\sum_{\substack{\left(x_{0}, y_{0}\right)_{p} \in \widetilde{\ell\left(S_{1}\right)} \\
\sum_{n=1}^{\infty}\left(x_{n}, y_{n}\right)_{p} \in \ell \widetilde{\sum_{n=2}^{\infty}} s_{n}}} P\left(\left(x_{0}, y_{0}\right)_{p}, \sum_{n=1}^{\infty}\left(x_{n}, y_{n}\right)_{p}\right) \log _{2}\left[P\left(\left(x_{0}, y_{0}\right)_{p}, \sum_{n=1}^{\infty}\left(x_{n}, y_{n}\right)_{p}\right)\right]
\end{aligned}
$$

Which means, we need $\mathscr{H}\left(\left(x_{0}, y_{0}\right)_{p}, \sum_{n=1}^{\infty}\left(x_{n}, y_{n}\right)_{p}\right)$ bits of information to establish its exact state, as we have the first hand knowledge of the base points $\left(x_{0}, y_{0}\right)_{p}$ the information that can be gained from $\left(x_{0}, y_{0}\right)_{p}$ is $\mathscr{H}\left(x_{0}, y_{0}\right)_{p}$ we need $\mathscr{H}\left(\left(x_{0}, y_{0}\right)_{p}, \sum_{n=1}^{\infty}\left(x_{n}, y_{n}\right)_{p}\right)-\mathscr{H}\left(x_{0}, y_{0}\right)_{p}$ bits to provide the system giving the chain relation of the entropy as; 


$$
\mathscr{H}\left(\sum_{n=1}^{\infty}\left(x_{n}, y_{n}\right)_{p} \mid\left(x_{0}, y_{0}\right)_{p}\right)=\mathscr{H}\left(\left(x_{0}, y_{0}\right)_{p}, \sum_{n=1}^{\infty}\left(x_{n}, y_{n}\right)_{p}\right)-\mathscr{H}\left(x_{0}, y_{0}\right)_{p}
$$

Therefore, the conditional entropy states the relation as an absolute equality [15];

$$
\begin{aligned}
& \mathscr{H}\left(\sum_{n=1}^{\infty}\left(x_{n}, y_{n}\right)_{p} \mid\left(x_{0}, y_{0}\right)_{p}\right) \\
& =\sum_{\left(x_{0}, y_{0}\right)_{p} \in \overline{\ell\left(S_{1}\right)}} P\left(\overline{\left(x_{0}, y_{0}\right)_{p}}, \overline{\sum_{n=1}^{\infty}\left(x_{n}, y_{n}\right)_{p}}\right) \log \frac{P\left(\overline{\left(x_{0}, y_{0}\right)_{p}}\right)}{P\left(\overline{\left(x_{0}, y_{0}\right)_{p}}, \overline{\left.\sum_{n=1}^{\infty}\left(x_{n}, y_{n}\right)_{p}\right)}\right.} \\
& \sum_{n=1}^{\infty}\left(x_{n}, y_{n}\right)_{p} \in \ell \overline{\sum_{n=2}^{\infty}} S_{n} \\
& =\sum_{\left(x_{0}, y_{0}\right)_{p} \in \overline{\ell\left(S_{1}\right)}} \log P\left(\overline{\left(x_{0}, y_{0}\right)_{p}}, \overline{\left.\sum_{n=1}^{\infty}\left(x_{n}, y_{n}\right)_{p}\right)}\right)\left(\log P\left(\overline{\left(x_{0}, y_{0}\right)_{p}}\right)\right. \\
& \sum_{n=1}^{\infty}\left(x_{n}, y_{n}\right)_{p} \in \ell \overline{\sum_{n=2}^{\infty}} S_{n} \\
& -\log P\left(\overline{\left(x_{0}, y_{0}\right)_{p}}, \overline{\left.\sum_{n=1}^{\infty}\left(x_{n}, y_{n}\right)_{p}\right)}\right) \\
& =-\sum_{\left(x_{0}, y_{0}\right)_{p} \in \overline{\ell\left(S_{1}\right)}} P\left(\overline{\left(x_{0}, y_{0}\right)_{p}}, \overline{\sum_{n=1}^{\infty}\left(x_{n}, y_{n}\right)_{p}}\right) \log \left(P\left(\left(\overline{\left(x_{0}, y_{0}\right)_{p}}, \overline{\sum_{n=1}^{\infty}}\left(x_{n}, y_{n}\right)_{p}\right)\right)\right) \\
& \sum_{n=1}^{\infty}\left(x_{n}, y_{n}\right)_{p} \in \ell \overline{\sum_{n=2}^{\infty}} S_{n} \\
& +\sum_{\left(x_{0}, y_{0}\right)_{p} \in \overline{\ell\left(S_{1}\right)}} P\left(\overline{\left(x_{0}, y_{0}\right)_{p}}, \overline{\left.\sum_{n=1}^{\infty}\left(x_{n}, y_{n}\right)_{p}\right)} \log \left(P\left(\overline{\left.\left(x_{0}, y_{0}\right)_{p}\right)}\right)=\mathscr{H}\left(\overline{\left(x_{0}, y_{0}\right)_{p}} \bar{\sum}_{n=1}^{\infty}\left(x_{n}, y_{n}\right)_{p}\right)\right.\right. \\
& \sum_{n=1}^{\infty}\left(x_{n}, y_{n}\right)_{p} \in \ell \widetilde{\sum_{n=2}^{\infty}} s_{n} \\
& +\sum_{\left(x_{0}, y_{0}\right)_{p} \in \overline{\ell\left(S_{1}\right)}} P\left(\overline{\left(x_{0}, y_{0}\right)_{p}}\right) \log \left(P\left(\overline{\left(x_{0}, y_{0}\right)_{p}}\right)\right)=\mathscr{H}\left(\left(x_{0}, y_{0}\right)_{p}, \sum_{n=1}^{\infty}\left(x_{n}, y_{n}\right)_{p}\right)-\mathscr{H}\left(x_{0}, y_{0}\right)_{p} \\
& \cong\left[\mathscr{H}\left(\left(x_{0}, y_{0}\right)_{p},\left(x_{1}, y_{1}\right)_{p} \ldots,\left(x_{n}, y_{n}\right)_{p}\right)=\sum_{i=1}^{n} \mathscr{H}\left(\left(x_{n}, y_{n}\right)_{p} \mid\left(x_{1}, y_{1}\right)_{p},\left(x_{2}, y_{2}\right)_{p}, \ldots,\left(x_{n-1}, y_{n-1}\right)_{p}\right)\right]
\end{aligned}
$$

Thus to provide the equivalence of the 5-tuple relation, we have;

$$
\begin{aligned}
& \sum_{\left(x_{0}, y_{0}\right)_{p} \in \overline{\ell\left(S_{1}\right)}} P\left(\overline{\left(x_{0}, y_{0}\right)_{p}}, \overline{\sum_{n=1}^{\infty}\left(x_{n}, y_{n}\right)_{p}}\right) \log \frac{P\left(\overline{\left(x_{0}, y_{0}\right)_{p}}\right)}{P\left(\overline{\left(x_{0}, y_{0}\right)_{p}}, \overline{\left.\sum_{n=1}^{\infty}\left(x_{n}, y_{n}\right)_{p}\right)}\right.} \\
& \sum_{n=1}^{\infty}\left(x_{n}, y_{n}\right)_{p} \in \ell \widetilde{\sum_{n=2}^{\infty}} s_{n} \\
& +\sum_{i=1}^{n} \mathscr{H}\left(\left(x_{n}, y_{n}\right)_{p} \mid\left(x_{1}, y_{1}\right)_{p},\left(x_{2}, y_{2}\right)_{p}, \ldots,\left(x_{n-1}, y_{n-1}\right)_{p}\right) \approx \operatorname{Det}\left[\begin{array}{ll}
\Psi^{+} \Phi^{+} & \Psi^{+} \Phi^{-} \\
\Psi^{-} \Phi^{+} & \Psi^{-} \Phi^{-}
\end{array}\right]+\ell\left(\sum_{n=2}^{\infty} S_{n}\right)
\end{aligned}
$$


For the equation $a+b \theta, \theta$ is the mutual information carrier between $a$ and $b$. Given, $a$ and $b$ random values, $\theta$ carries the mutual dependency between them which is the amount of information that can be obtained from one random variables by imposing authority on the other variables, its not necessary that the variables stick to two, it can be upto infinity but as we state earlier the variables are amplifiers where one variable amplifies the other like in the equation $a+c+b \theta, c$ amplifies $a$ and $b$ while $\theta$ coordinates the information between them, which is linked to the 'entropy' where information of the random variable is quantized. Now, in the Kullback-Leibler [16] divergence notation $D_{K L}$ this can be defined over the function of the entropic space (or curve) $\widetilde{\ell\left(S_{1}\right)} \times \ell \widetilde{\sum_{n=2}^{\infty}} S_{n}$ of a pair of the points, $\left(x_{0}, y_{0}\right)_{p} \times \sum_{n=1}^{\infty}\left(x_{n}, y_{n}\right)_{p}$ with the joint distribution $P_{\left(\left(x_{0}, y_{0}\right)_{p}, \sum_{n=1}^{\infty}\left(x_{n}, y_{n}\right)_{p}\right)}^{J}$ having the marginal distribution $P_{\left(\left(x_{0}, y_{0}\right)_{p}\right)}^{M}$ and $P_{\left(\sum_{n=1}^{\infty}\left(x_{n}, y_{n}\right)_{p}\right)}^{M}$ the mutual information can be denoted by the relation with $\theta$ replacing $I$ is;

$$
\theta\left(\left(x_{0}, y_{0}\right)_{p}, \sum_{n=1}^{\infty}\left(x_{n}, y_{n}\right)_{p}\right)=D_{K L}\left(P_{\left(\left(x_{0}, y_{0}\right)_{p}, \Sigma_{n=1}^{\infty}\left(x_{n}, y_{n}\right)_{p}\right)}^{J} \| P_{\left(\left(x_{0}, y_{0}\right)_{p}\right)}^{M} \bigotimes P_{\left(\sum_{n=1}^{\infty}\left(x_{n}, y_{n}\right)_{p}\right)}^{M}\right)
$$

Therefore, the mutual information can be stated in a formal way keeping in mind $\theta\left(\left(x_{0}, y_{0}\right)_{p}, \sum_{n=1}^{\infty}\left(x_{n}, y_{n}\right)_{p}\right) \leq$ $\mathscr{H}\left(x_{0}, y_{0}\right)_{p}$ by the relation $[17]$

$$
\begin{aligned}
& \theta\left(\left(x_{0}, y_{0}\right)_{p}, \sum_{n=1}^{\infty}\left(x_{n}, y_{n}\right)_{p}\right) \\
& =\sum_{\left(x_{0}, y_{0}\right)_{p} \in \overline{\ell\left(S_{1}\right)}} \sum_{\sum_{n=1}^{\infty}\left(x_{n}, y_{n}\right)_{p} \in \ell} P_{\left(\left(x_{0}, y_{0}\right)_{p}, \sum_{n=2}^{\infty} S_{n}\left(x_{n}, y_{n}\right)_{p}\right)}\left(\overline{\left(x_{0}, y_{0}\right)_{p}}, \overline{\sum_{n=1}^{\infty}\left(x_{n}, y_{n}\right)_{p}}\right) \\
& \times \log \left(\frac{P_{\left(\left(x_{0}, y_{0}\right)_{p}, \sum_{n=1}^{\infty}\left(x_{n}, y_{n}\right)_{p}\right)}\left(\overline{\left(x_{0}, y_{0}\right)_{p}}, \overline{\sum_{n=1}^{\infty}\left(x_{n}, y_{n}\right)_{p}}\right)}{\left(P_{\left(x_{0}, y_{0}\right)_{p}} \overline{\left(x_{0}, y_{0}\right)_{p}}\right)\left(P_{\sum_{n=1}^{\infty}\left(x_{n}, y_{n}\right)_{p}} \overline{\sum_{n=1}^{\infty}\left(x_{n}, y_{n}\right)_{p}}\right)}\right) \\
& =\mathscr{H}\left(\left(x_{0}, y_{0}\right)_{p}, \sum_{n=1}^{\infty}\left(x_{n}, y_{n}\right)_{p}\right)-\mathscr{H}\left(\left(x_{0}, y_{0}\right)_{p}\right)-\mathscr{H}\left(\sum_{n=1}^{\infty}\left(x_{n}, y_{n}\right)_{p}\right)
\end{aligned}
$$

Discussions - Through the principle of the incomplete elliptic integral of the $2^{\text {nd }}$ kind, this has been deliberately proved that, the system can conserve entropy by the principle of the equivalence closure provided entropy of the initial state which has been known, gives rise to the next state from where the conditional 
entropy takes place and gets conserved by means of the mutual information carriers, that is, $\theta$ making a way out for the sets to close equivalently via equivalence closure theorem to guide the elaborate process of the evolving dynamical system over repeated iterations in a conserved means through a local attractor. More emphasis has been made with respect of the mean and the support set, where through the summation convention, the relation of equivalence closure has been properly diversified by the spinor and spreader matrix determinants in the crucial base points over repeated revolutions around it. This process can run infinitely with a more probabilistic amplitude of the correct divergences that loops around the base points like its previous thereby maintaining the property of the conservative (more precisely ergodic) systems in a 5-tuple measure space through the generators of the space.

Declaration: Author of this paper has no conflicting interests.

Funding - No funding has been issued for the purpose of this work.

\section{Reference -}

[1] THOMSEN, K. (2006). On the ergodic theory of synchronized systems. Ergodic Theory and Dynamical Systems, 26(04), 1235. https://doi.org/10.1017/s0143385706000290

[2] Ornstein, D. (1971). A remark on the Birkhoff ergodic theorem. Illinois Journal of Mathematics, 15(1). https://doi.org/10.1215/ijm/1256052821

3] Birkhoff, G. D. (1931). Proof of the Ergodic Theorem. Proceedings of the National Academy of Sciences, 17(12), 656-660. https://doi.org/10.1073/pnas.17.2.656

[4] Joot, P. (2013, February 5). Liouville's theorem questions on density and current. Peeter Joot's (OLD) Blog. https://peeterjoot.wordpress.com/2013/02/05/liouvilles-theorem-questions-on-densityand-current/

[5] Mehling, K. (2020). Introduction To Ergodic Theory With Applications To Physics. Chicago Math Lectures. Published. 
[6] ROY, E. (2009). Poisson suspensions and infinite ergodic theory. Ergodic Theory and Dynamical Systems, 29(2), 667-683. https://doi.org/10.1017/s0143385708080279

[7] Incomplete elliptic integral of the 2nd kind $\mathrm{E}(\varphi, \mathrm{k})$ Calculator. (2021). High Accuracy Calculation for Life or Science. https://keisan.casio.com/exec/system/1244989948

[8] BENOIST, Y., \& OH, H. (2021). Geodesic planes in geometrically finite acylindrical -manifolds. Ergodic Theory and Dynamical Systems, 1-40. https://doi.org/10.1017/etds.2021.19

[9] ZANG, Y. (2021). Entropies and volume growth of unstable manifolds. Ergodic Theory and Dynamical Systems, 1-15. https://doi.org/10.1017/etds.2021.2

[10] Karamanos, K., Mistakidis, I., \& Mistakidis, S. (2012). The many facets of Poincare recurrence theorem of the logistic map. Kybernetes, 41(5/6), 794-803. https://doi.org/10.1108/03684921211243428

[11] Nourdin, I., Peccati, G., \& Seuret, S. (2020). Sojourn time dimensions of fractional Brownian motion. Bernoulli, 26(3). https://doi.org/10.3150/19-bej1105

[12] Fischer, I. (2020). The Conditional Entropy Bottleneck. Entropy, 22(9), 999. https://doi.org/10.3390/e22090999

[13] Gokhale, D. (1999). On Joint and Conditional Entropies. Entropy, 1(2), 21-24. https://doi.org/10.3390/e1020021

[14] Luo, L., Wang, J., Zhang, L., \& Zhang, S. (2016). The Differential Entropy of the Joint Distribution of Eigenvalues of Random Density Matrices. Entropy, 18(9), 342. https://doi.org/10.3390/e18090342

[15] Phoenix, S. (1992). Elements of Information Theory. Journal of Modern Optics, 39(7), 1600-1601. https://doi.org/10.1080/09500349214551641

[16] Belov, D. I., \& Armstrong, R. D. (2011). Distributions of the Kullback-Leibler divergence with applications. British Journal of Mathematical and Statistical Psychology, 64(2), 291-309. https://doi.org/10.1348/000711010x522227

[17] Ben-Naim, A. (2019). Entropy and Information Theory: Uses and Misuses. Entropy, 21(12), 1170. https://doi.org/10.3390/e21121170 